\title{
Protein levels of tissue inhibitor of metalloproteinase- 1 in tumor extracts as a marker for prognosis and recurrence in patients with gastric cancer
}

\author{
Takaki Yoshikawa ${ }^{1}$, Akira Tsuburaya ${ }^{1}$, Osamu Kobayashi ${ }^{1}$, Motonori Sairenji ${ }^{1}$, and Yohei Miyagi ${ }^{2}$ \\ ${ }^{1}$ Department of Gastrointestinal Surgery, Kanagawa Cancer Center, Yokohama, Japan \\ ${ }^{2}$ Department of Molecular Pathology, Kanagawa Cancer Center, 1-1-2 Nakao, Asahi-ku, Yokohama 241-0815, Japan
}

\begin{abstract}
Background. Tissue inhibitor of metalloproteinase-1 (TIMP1) correlates with tumor progression in patients with gastric cancer; however, the clinical significance of TIMP-1 as a marker for prognosis and recurrence has not been fully clarified.

Methods. TIMP-1 protein was measured by an enzyme-linked immunosorbent assay in tumor samples from 86 patients who had undergone surgical resection. An intratumoral TIMP-1 value of $10.0 \mathrm{ng} / \mathrm{mg}$ protein or more was defined as positive. Patients were followed up for more than 5 years prospectively. Results. Thirty-one of the 86 patients $(36.0 \%)$ were positive for TIMP-1. Kaplan-Meier curves for overall survival were significantly different between patients who were positive and those who were negative for TIMP-1. Univariate analysis of factors affecting overall survival showed that depth of tumor invasion; lymph node metastasis; peritoneal dissemination; lymphatic invasion; venous invasion; Lauren classification of histology; curability; and TIMP-1 were statistically significant. Stepwise multivariate analysis for overall survival demonstrated that depth of tumor invasion, nodal metastasis, peritoneal dissemination, and TIMP-1 remained independent prognostic factors. Kaplan-Meier curves for disease-free survival were significantly different between patients who were positive and those who were negative for TIMP-1. The incidence of recurrence was significantly higher in patients positive for TIMP-1 than in those who were negative for TIMP-1. The frequency at each site of recurrence was higher in patients positive for TIMP-1.

Conclusion. These results suggested that the protein concentration of TIMP-1 in the tumor extracts was a useful marker for overall survival, disease-free survival, and disease recurrence in patients with gastric cancer. Thus, tumor TIMP-1 may serve to identify a high-risk group, for whom optimal surgical and medical treatment can be given.
\end{abstract}

Key words TIMP-1 - Gastric carcinoma - Prognostic value . Recurrence

Offprint requests to: T. Yoshikawa

Received: October 21, 2005 / Accepted: January 16, 2006

\section{Background}

Gastric cancer is the most frequent malignancy in Japan [1] and the second leading cause of death in the world [2]. Although the mortality has gradually decreased with an increasing incidence of early-stage disease in Japan, more than $50 \%$ of advanced cases recur, even after curative resection [3]. Prognosis is dismal in patients with recurrent or unresectable gastric cancer, and their median survival time is less than 1 year, even if they receive chemotherapy [4]. Therefore, reliable biomarkers to predict relapse and prognosis are much needed to optimize surgical and medical management for a high risk group.

Recent advances in molecular biology have revealed some mechanisms involved in tumor progression. The family of matrix metalloproteinases (MMPs) plays a significant role in matrix degradation which is an essential step in tumor invasion and metastasis [5,6]. These proteinases are produced not only by tumor cells but also by stromal cells [5-9]. In addition to these proteinases, the tissue inhibitors of metalloproteinases (TIMPs) play an important role in the extent of matrix degradation [5-8]. Once MMPs are activated, the synthesis of TIMPs is induced in stromal cells in response to the proteinase reactions [6]. The TIMP family currently consists of four members: TIMP-1, TIMP-2, TIMP-3, and TIMP-4 [7]. Of these known TIMPs, TIMP-1 strongly inhibits various types of MMPs [7-9]. In addition to its inhibitory properties, TIMP-1 has additional activities, such as growth stimulation [10-13] and the inhibition of apoptosis $[14,15]$ in carcinoma cells.

Although these observations suggest that TIMP-1 is important in stimulating tumor growth, the clinical role of TIMP-1 in terms of its prognostic value remains largely unknown in patients with gastric cancer. Previously, Nomura et al. [16] and Murray et al. [17] reported on the expression of TIMP-1 protein in gastric tumors. Mimori et al. [18] observed a positive correlation 
between elevated TIMP-1 mRNA concentrations and tumor progression. Recently, we [19] found that high levels of TIMP-1 protein in tumor extracts correlated with tumor aggressiveness in patients with gastric cancer. In that study, we [19] also showed, by analyzing the short-term survival in these patients, that tumor TIMP-1 could be a candidate prognostic factor [19]. Based on these findings, the present study was conducted to further clarify by analyzing the complete 5year follow-up observations, the clinical significance of tumor TIMP-1 as a marker of prognosis and recurrence in patients with gastric cancer.

\section{Patients and methods}

\section{Patients}

Tumor samples were obtained from 86 patients who had undergone surgical resection for gastric cancer at the Kanagawa Cancer Center (Yokohama, Japan) between August 1996 and March 1998. None of the patients had undergone any prior treatment. Tumor classification and pathologic diagnosis were conducted according to the Japanese classification of gastric carcinoma [20].

\section{Preparation tissue samples}

Tissue samples were immediately frozen after excision and stored at $-80^{\circ} \mathrm{C}$ until processed. Frozen tissue samples (200 mg each) were homogenized in phosphatebuffered saline (PBS) and centrifuged at $8000 \mathrm{~g}$ for 20 min. Supernatants were then assayed for TIMP-1 (description of assay follows). The DC protein assay (Bio-Rad, Hercules, CA, USA) was used to measure the total protein concentration of each sample.

\section{Measurement of TIMP-1 protein}

A one-step sandwich enzyme-linked immunosorbent assay (ELISA) kit (Fuji Chemical, Takaoka, Japan) was used to measure the protein concentration of TIMP-1 [21,22]. Serially diluted human TIMP-1 standards and $5 \mu$ of each properly diluted tumor sample homogenate were added to separate wells of a 96-well plate, together with $75 \mu \mathrm{l}$ of anti-human TIMP-1 antibody conjugated to peroxidase. Samples were incubated for $30 \mathrm{~min}$ at $30^{\circ} \mathrm{C}$, and then washed with PBS supplemented with $0.05 \%(\mathrm{v} / \mathrm{v})$ Tween 20 . The peroxidase reaction was carried out in an $\mathrm{H}_{2} \mathrm{O}_{2}$ solution containing $O$ phenylenediamine, a colorimetric marker, at $30^{\circ} \mathrm{C}$ for $15 \mathrm{~min}$, and was stopped by adding $\mathrm{H}_{2} \mathrm{SO}_{4}$. A microplate reader was used to measure absorbance at $492 \mathrm{~nm}$. The sensitivity limit of the assay was $50 \mathrm{ng} / \mathrm{ml}$ and the assay was linear from 51 to $2000 \mathrm{ng} / \mathrm{ml}$. The coefficient of variation was less than 5.0\%. TIMP-1 concentrations were expressed as nanograms TIMP-1 per milligram of total protein in each supernatant. The cutoff value for tumor TIMP-1 protein was set at $10 \mathrm{ng}$ TIMP-1/mg protein, based on the results of our previous study [19]. Tumor TIMP-1 concentrations of $10 \mathrm{ng} / \mathrm{mg}$ protein or more were defined as positive [19].

\section{Follow-up examinations}

After curative surgery, patients were examined at the outpatient clinic every 3 months for the first 3 years, and then every 6 months for the subsequent 2 years. The patients were physically examined and assessed for serum levels of the tumor markers carcinoembryonic antigen (CEA) and carbohydrate antigen (CA) 19-9 at each examination, and underwent computed tomography (CT) examinations every year. When the physical examinations or tumor marker results suggested a recurrence of cancer, the patients underwent CT, barium enema, and/or diagnostic laparoscopy.

\section{Medical treatment}

Patients received chemotherapy including 5-fluorouracil after noncurative surgery and after recurrence of the disease when chemotherapy was feasible. Best supportive care was selected when the tumor did not respond to chemotherapy or when chemotherapy was not appropriate because of the patient's poor condition.

\section{Statistical analyses}

Either an unpaired Student's $t$-test or a $\chi^{2}$ test was used to compare results between the TIMP-1-positive and -negative groups. The survival curves were calculated using the Kaplan-Meier method and compared by the log-rank test. Cox's proportional hazard model was used to perform univariate and multivariate survival analyses. A $P$ value of $<0.05$ was defined as statistically significant, and the data values were expressed as means \pm SDs. SPSS software (v11.0J Win, SPSS, Chicago, IL, USA) was used for all statistical tests.

\section{Results}

Positive tumor TIMP-1 was observed in 31 of the 86 patients $(36.0 \%)$. When clinicopathologic factors were compared between patients positive and those negative for TIMP-1, significant differences were observed in depth of tumor invasion, lymphatic invasion, and tumor stage (Table 1).

All 86 patients were followed for more than 5 years and the outcomes were obtained through clinical ap- 
Table 1. Background of patients

\begin{tabular}{|c|c|c|c|}
\hline & $\begin{array}{l}\text { TIMP-1 negative } \\
\quad(n=55)\end{array}$ & $\begin{array}{l}\text { TIMP-1 positive } \\
\quad(n=31)\end{array}$ & $P$ value \\
\hline Sex; male/female & $37 / 18$ & $22 / 9$ & 0.723 \\
\hline Age (years) & $60.0 \pm 10.6$ & $63.2 \pm 8.6$ & 0.148 \\
\hline \multicolumn{4}{|l|}{ Macroscopic type } \\
\hline T1 Type 0 & 17 & 0 & NA \\
\hline T2 Type 1 & 3 & 2 & 0.552 \\
\hline Type 2 & 4 & 6 & \\
\hline Type 3 & 7 & 9 & \\
\hline Type 4 & 10 & 7 & \\
\hline Type 5 & 14 & 7 & \\
\hline Depth of invasion & & & 0.002 \\
\hline $\mathrm{T} 1$ & 17 & 0 & \\
\hline $\mathrm{T} 2$ & 13 & 8 & \\
\hline $\mathrm{T} 3$ & 25 & 23 & \\
\hline Histological type & & & 0.121 \\
\hline Intestinal & 22 & 7 & \\
\hline Diffuse & 33 & 23 & \\
\hline Lymph node metastasis & & & 0.264 \\
\hline No & 21 & 6 & \\
\hline N1 & 14 & 12 & \\
\hline $\mathrm{N} 2$ & 13 & 7 & \\
\hline N3 & 7 & 6 & \\
\hline Peritoneal metastasis & & & 0.658 \\
\hline Negative & 44 & 26 & \\
\hline Positive & 11 & 5 & \\
\hline Liver metastasis & & & NA \\
\hline Negative & 55 & 31 & \\
\hline Lymphatic invasion & & & 0.003 \\
\hline Negative & 25 & 4 & \\
\hline Positive & 30 & 27 & \\
\hline Venous invasion & & & 0.661 \\
\hline Negative & 36 & 19 & \\
\hline Positive & 19 & 12 & \\
\hline Curability & & & 0.810 \\
\hline $\mathrm{AB}$ & 45 & 26 & \\
\hline $\mathrm{C}$ & 10 & 5 & \\
\hline Stage & & & \\
\hline I & 24 & 2 & 0.002 \\
\hline II & 4 & 7 & \\
\hline III & 14 & 14 & \\
\hline IV & 13 & 8 & \\
\hline
\end{tabular}

NA, not applicable

a Unpaired Student's $t$-test or the $\chi^{2}$ method was used to compare the data between the two groups

pointments at the outpatient clinic or by telephone contact. The median observation period was 60.1 months (range, 60.0-69.4 months). Of the 86 patients, 45 died during the follow-up period; 15 were in the TIMP-1negative group and 30 in the TIMP-1 positive group. Kaplan-Meier curves for overall survival were significantly different between patients who were positive and those who were negative for TIMP-1 (Fig. 1). Overall survival rates were $65.5 \%$ at 3 years and $58.2 \%$ at 5 years in patients negative for TIMP-1, while these values were $41.9 \%$ at 3 years and $35.5 \%$ at 5 years in patients positive for TIMP-1. Overall survival was compared between patients who were negative and those who were positive for TIMP-1, stratified by disease stage (Table 2). Overall survival rates were lower in the positive TIMP-1 group with stage II/stage III than in the negative group with stage II/III, although the difference did not reach significance. Univariate analysis of factors affecting overall survival demonstrated that depth of tumor invasion; lymph node metastasis; peritoneal dissemination; lymphatic invasion; venous invasion; Lauren classification of histology; curability; and TIMP1 were statistically significant (Table 3 ). Stepwise multivariate analysis for overall survival revealed that depth of tumor invasion, metastasis to lymph nodes, peritoneal dissemination, and TIMP-1 remained independent 


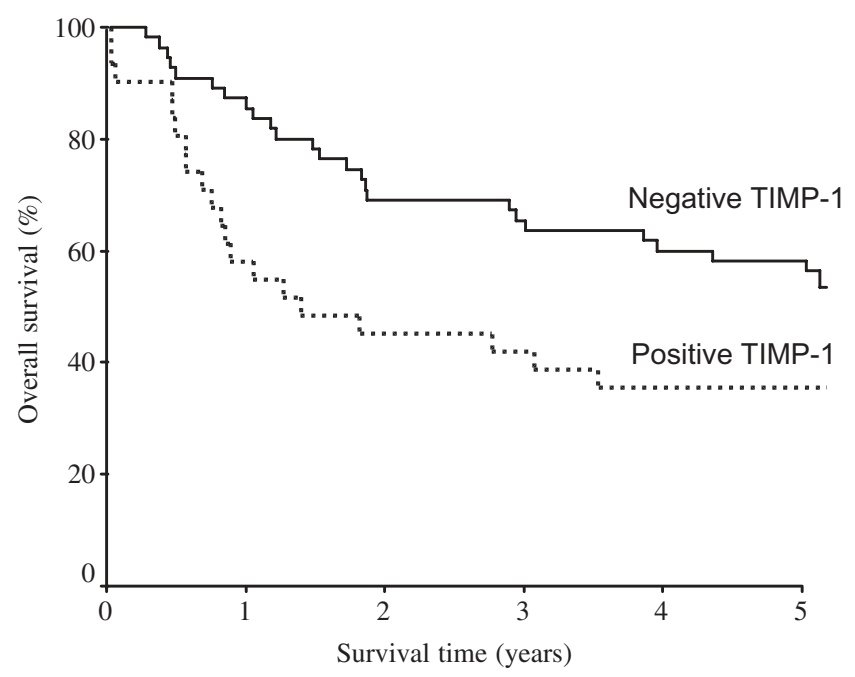

Fig. 1. Kaplan-Meier curves for overall survival showed a significant difference between patients who were negative (solid line) and those who were positive (broken line) for tumor tissue inhibitor of metalloproteinase-1 (TIMP-1) $(P=$ 0.0097) prognostic factors, although the statistics for depth of tumor invasion, nodal metastases, and TIMP-1 were marginally significant (Table 4).

The disease recurred in 22 of the 71 patients who underwent curative resection among the 86 patients. Kaplan-Meier curves for disease-free survival were significantly different between patients who were positive and those who were negative for TIMP-1 (Fig. 2). The incidence of recurrence was significantly higher in patients positive for TIMP-1 than in those negative for TIMP-1 (Table 5). The frequency at each site of recurrence was higher in patients positive for TIMP-1; however, there was no significant difference in the recurrent patterns (Table 6).

\section{Discussion}

This study indicated that gastric cancer patients who were positive for intratumoral TIMP-1 had a poor prognosis. Moreover, multivariate analysis revealed that

Table 2. Survival rates of patients negative and those positive for TIMP-1, stratified by stage of disease

\begin{tabular}{lrrrrrrrr}
\hline & \multicolumn{3}{c}{ Negative for TIMP-1 } & & \multicolumn{3}{c}{ Positive for TIMP-1 } & \\
\cline { 2 - 3 } Stage & No. & 3-Year & 5 5-Year & & No. & 3-Year & 5-Year & $P$ value \\
\hline I & 24 & $91.7 \%$ & $87.5 \%$ & & 2 & $100 \%$ & $100 \%$ & 0.556 \\
II & 4 & $100 \%$ & $100 \%$ & & 7 & $57.1 \%$ & $57.1 \%$ & 0.154 \\
III & 14 & $64.3 \%$ & $50 \%$ & & 14 & $42.9 \%$ & $35.7 \%$ & 0.379 \\
IV & 13 & $7.7 \%$ & $0 \%$ & & 8 & $12.5 \%$ & $0 \%$ & 0.341 \\
II/III & 18 & $72.2 \%$ & $61.1 \%$ & & 21 & $47.6 \%$ & $42.9 \%$ & 0.238 \\
\hline
\end{tabular}

Table 3. Univariate Cox proportional hazards analysis of clinicopathologic factors

\begin{tabular}{llcc}
\hline Factors (category) & No. of patients & $P$ value & Hazard ratio \\
\hline Depth of invasion (T1 vs T2 vs T3-T4) & 17,21 , and 48 & 0.000 & 3.327 \\
Lymph node metastasis (N0-1 vs N2-4) & 53 and 33 & 0.000 & 5.001 \\
Peritoneal metastasis (negative vs positive) & 70 and 16 & 0.000 & 5.109 \\
Lymphatic invasion (negative vs positive) & 28 and 57 & 0.005 & 3.030 \\
Venous invasion (negative vs positive) & 53 and 31 & 0.049 & 1.829 \\
Lauren classification (intestinal vs diffuse) & 29 and 56 & 0.040 & 2.038 \\
Curability (AB vs C) & 71 and 15 & 0.000 & 5.652 \\
TIMP-1 (negative vs positive) & 55 and 31 & 0.027 & 1.943 \\
\hline
\end{tabular}

Table 4. Stepwise multivariate Cox proportional hazards analysis of clinicopathologic factors

\begin{tabular}{llcc}
\hline Factors (category) & No. of patients & $P$ value & Hazard ratio \\
\hline Depth of invasion (T1 vs T2 vs T3-T4) & 17,21 , and 48 & 0.061 & 1.926 \\
Lymph node metastasis (N0-1 vs N2-4) & 53 and 33 & 0.069 & 2.052 \\
Peritoneal metastasis (negative vs positive) & 70 and 16 & 0.010 & 2.819 \\
TIMP-1 (negative vs positive) & 55 and 31 & 0.083 & 1.798 \\
\hline
\end{tabular}


tumor TIMP-1 was one of the independent prognostic factors for overall survival. These data suggested that the protein level of TIMP-1 in tumor extracts was a useful prognostic marker in patients with gastric cancer. Previously, we demonstrated that intratumoral TIMP-1 concentration was an independent prognostic factor for the short-term survival of patients with gastric carcinoma [19]. In that study, however, the observation period ranged from 350 days to 650 days, which was too short for a definitive conclusion on the prognostic value. In the present study, all patients were followed for more than 5 years. This report finally confirmed that tumor

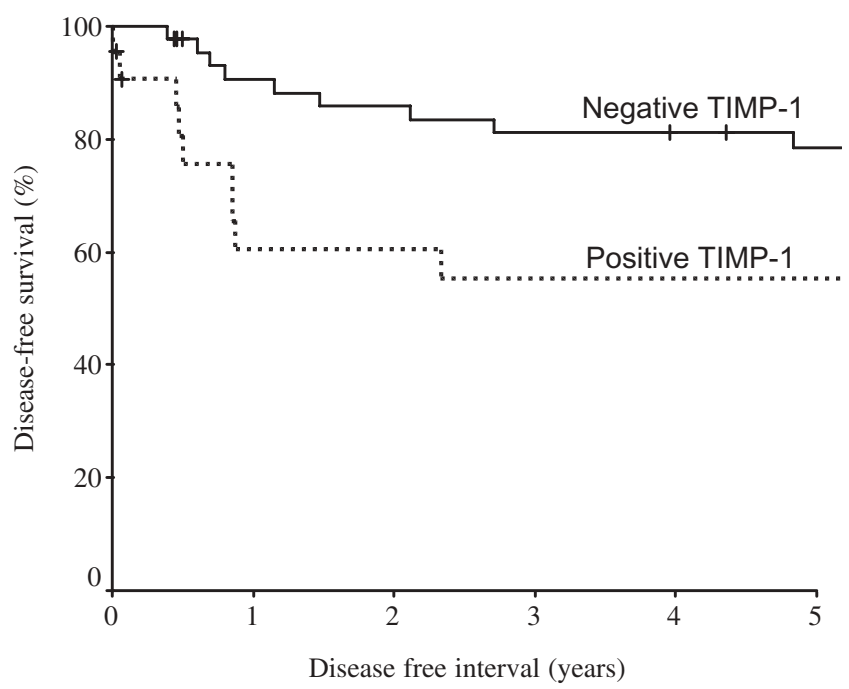

Fig. 2. Kaplan-Meier curves for disease-free survival indicated that the difference was statistically significant between patients who were negative (solid line) and those who were positive (broken line) for tumor TIMP-1 $(P=0.03)$. Censored cases were deaths due to causes other than cancer, such as traffic accident, stroke, and myocardial infarction

Table 5. Intratumoral TIMP-1 concentration and disease recurrence in patients who underwent curative resection

\begin{tabular}{lcc}
\hline Recurrence & TIMP-1 negative & TIMP-1 positive \\
\hline Negative & 36 & 13 \\
Positive & 9 & 13
\end{tabular}

$P=0.008$
TIMP-1 was an independent prognostic factor for overall survival in patients with gastric cancer.

In the present study, a cutoff value of $10 \mathrm{ng} / \mathrm{mg}$ protein for the protein level of TIMP-1 in the tumor extracts was determined using the upper value observed in T1 tumors, because MMPs and TIMPs would be less likely to be induced in these tumors, as degradation of the matrix is not initiated in early disease, and because the prognosis of early gastric cancer is excellent [23]. In fact, Nomura et al. [16] reported that MMP-2 was not elevated in patients with early gastric cancer. Mimori et al. [18] also demonstrated that TIMP-1 mRNA was not increased in early gastric cancer. Although other methods of defining a cutoff value can be used, the value we set clearly separated patients with good and bad prognoses.

Apart from our previous study [19], there has been only one other report (Schrohl et al. [24]) of TIMP-1 measurements in tumor extracts done by ELISA. They demonstrated that intratumoral TIMP-1 level was an independent prognostic factor for patients with breast cancer, in a study that was performed as a European Organization for Research and Treatment of Cancer Receptor and Biomarker Group collaborative study [24]; their cutoff value was set at $11.71 \mathrm{ng} / \mathrm{mg}$ protein, using isotonic regression analysis. However, the prognostic significance of TIMP-1 has not been confirmed in other types of malignancies. In the present study, we have, for the first time, demonstrated the prognostic value of tumor TIMP-1 concentrations in patients with gastric cancer. A few investigators have, however, examined the production of TIMP-1 in the tumor tissue of gastric cancer by immunohistochemical analyses [16,25]. Joo et al. [25] demonstrated that TIMP-1 was detected predominantly in the peritumoral stromal cells rather than the tumor cells themselves. Moreover, they reported that the intensity of TIMP-1 immunohistochemical staining correlated positively with tumor stage and survival [25]. Recently, enhanced immunostaining of TIMP-1 has been reported to be correlated with prognosis in patients with urothelial [26], renal cell [27], and non-small-cell lung cancers [28]. Immunohistochemical analyses are convenient and suitable for examining stored samples retrospectively, but the intensity of immunostaining is decreased over time

Table 6. Site of disease recurrence classified by intratumoral TIMP-1 concentration

\begin{tabular}{lcc}
\hline Site of recurrence & TIMP-1 negative $(\%$ of total cases) & TIMP-1 positive (\% of total cases) \\
\hline Peritoneum & $6 / 45(13.3 \%)$ & $7 / 26(26.9 \%)$ \\
Liver & $0 / 45$ & $1 / 26(3.8 \%)$ \\
Lymph nodes & $3 / 45(6.7 \%)$ & $4 / 26(15.4 \%)$ \\
Local & $0 / 45$ & $1 / 26(3.8 \%)$
\end{tabular}

$P=0.672$ 
because of loss of sensitivity secondary to antigenic alterations caused by fixation procedures and the storage of the samples. Immunohistochemical evaluation is objective, but not quantitative or reproducible. It has been reported that elevated levels of TIMP-1 mRNA were associated with poor prognosis in patients with some other tumors, including breast [29], esophageal [30], and non-small-cell lung cancers [31]. The presence of TIMP1 has also been shown in body fluids (including plasma and urine) in various types of malignancies, including those of the rectum, colon, ovary, and bladder, and in lung cancer [32-37]. To date, we have reported that TIMP-1 was detected in the plasma, and was correlated with tumor progression and survival in patients with gastric and colorectal cancers [38-41].

The finding that TIMP-1 was correlated with poor prognosis seemed surprising, given that TIMP-1 inhibits MMPs, which play a major role in tissue degradation by tumor cells. However, additional activities of TIMP-1 have been reported: stimulating growth in several cell types [10-13] and inhibiting apoptosis [14,15]. Both such activities could increase proliferation and induce the tumor growth. More recently, a regulatory role in angiogenesis has been suggested for TIMP-1 $[42,43]$. In our study, comparison of clinicopathologic factors between patients who were positive and those who were negative for TIMP-1 revealed a significant correlation with depth of tumor invasion, lymphatic invasion, and stage of disease. Thus, TIMP-1 may play a role in tumor aggressiveness, through the stimulation of cancer cell growth, shortening the survival of patients with gastric cancer.

Generally, in patients with gastric cancer, the most important factors affecting survival have been found to be depth of tumor invasion, lymph node metastasis, and the presence of distant metastases to the peritoneum or the liver. Measurement of the tumor tissue concentration of TIMP-1 has a great advantage when compared with other prognostic factors. Intratumoral TIMP-1 can be determined by assaying biopsy specimens before surgery, which could provide potentially useful prognostic information that can aid in formulating an effective treatment strategy, whereas depth of invasion and lymph node metastases are determined pathologically after surgical resection. For example, if a patient has a gastric tumor that can be treated with surgery, but the tumor has a high intratumoral TIMP-1 protein concentration, pre- or postoperative chemotherapy can be used to attempt to improve the prognosis.

In the present study, multivariate analysis revealed that the statistics for TIMP-1, as well as those for depth of tumor invasion and nodal metastasis, were marginally significant as prognostic factors. Subset analyses of overall survival rates stratified by tumor stage demonstrated that there was some difference between those positive for TIMP-1 and those negative for TIMP-1 in stages II and stage III, although these statistics too did not reach significance for prognosis. The number of patients examined in this study was too small to reach a definite conclusion on the statistical significance of TIMP-1 as a prognostic factor. Further study will clarify the prognostic value of TIMP-1 in patients with gastric cancer by targeting patients with cancers at stages II and III and using an appropriate sample size to confirm statistical significance.

In patients with gastric cancer, the most frequent sites of recurrence are the peritoneum, lymph node, liver, and local sites [44]. To date, the association between TIMP-1 and disease-free survival or recurrence patterns has not been examined in patients with cancers. TIMP1 promotes cell proliferation [10-13] and inhibits apoptosis [14,15], actions which may increase tumor aggressiveness and enhance metastases nonspecifically, while TIMP-1 also plays a role in angiogenesis $[42,43]$, which may augment hematological metastases. In the present study, the rate of disease-free survival was significantly lower in patients who were positive for TIMP1 than in those who were negative for TIMP-1; moreover, the incidence of recurrence was significantly higher in patients positive for TIMP-1. Although the frequency at each site of recurrence was higher in patients positive for TIMP-1, the distribution of the recurrence patterns was not significantly different. These data may suggest that TIMP-1 could be a marker for disease recurrence and disease-free survival in patients with gastric cancer. TIMP-1 may enhance metastases nonspecifically.

In conclusion, our results suggested that the protein concentration of TIMP-1 in tumor extracts was a useful marker for overall survival, disease-free survival, and disease recurrence in patients with gastric cancer. Thus, tumor TIMP-1 may serve to identify a high-risk group, for whom optimal surgical and medical treatment can be given.

Acknowledgments This work was supported in part by a Grant-in-Aid for Cancer Research (10-27) from the Ministry of Health and Welfare of Japan.

\section{References}

1. The Research Group for Population-based Cancer Registration in Japan. Cancer incidence in Japan, 1985-89: re-estimation based on data from eight population-based cancer registries. Jpn J Clin Oncol 1998;28:54-67.

2. Pisani P, Parkin DM, Bray F, Ferlay J. Estimates of the worldwide mortality from 25 cancers in 1990. Int J Cancer 1999;83:18-29.

3. Nakajima T, Ohta K, Ohyama K. Tabular analysisi of CIH (Cancer Institute Hospital) database. In: Nakajima T, Yamaguchi T, editors. Multimodality therapy for gastric cancer. Berlin Heidelberg New York Tokyo: Springer Verlag; 1999. p 197-267. 
4. Wilke HJ, Van Cutsem E. Current treatments and future perspectives in colorectal and gastric cancer. Ann Oncol 2003;14: 49-55.

5. Stamenkovic I. Matrix metalloproteinases in tumor invasion and metastasis. Semin Cancer Biol 2000;10:415-33.

6. Curran S, Murray GI. Matrix metalloproteinases in tumour invasion and metastasis. J Pathol 1999;189:300-8.

7. Gomez DE, Alonso DF, Yoshiji H, Thorgeirsson UP. Tissue inhibitors of metalloproteinases: structure, regulation and biological functions. Eur J Cell Biol 1997;74:111-22.

8. Crawford HC, Matrisian LM. Tumor and stromal expression of matrix metalloproteinases and their role in tumor progression. Invasion Metastasis 1995;14:234-45.

9. Denhardt TD, Feng Bo, Edwards DR, Cocuzzi ET, Malyankar UM. Tissue inhibitor of metalloproteinase (TIMP, aka EPA): structure, control of expression and biological functions. Pharmacol Ther 1993;59:329-41.

10. Docherty AJ, Lyons A, Smith BJ, Wright EM, Stephens PE, Harris TJ, et al. Sequence of human tissue inhibitor of metalloproteinases and its identity to erythroid-potentiating activity. Nature 1985;318:66-9.

11. Hayakawa H, Yamashita K, Ohwaki K, Sawa M, Noguchi T, Iwata $\mathrm{K}$, et al. Collagenase activity and tissue inhibitor of metalloproteinases-1 (TIMP-1) content in human whole saliva from clinically healthy and periodontally diseased subjects. J Periodontal Res 1994;29:305-8.

12. Chesler L, Golde DW, Bersch N, Johnson MD. Metalloproteinase inhibition and erythroid potentiation are independent activities of tissue inhibitor of metalloproteinases-1. Blood 1995;86: 4506-15.

13. Luparello C, Avanzato G, Carella C, Pucci-Minafra I. Tissue inhibitor of metalloprotease (TIMP)-1 and proliferative behaviour of clonal breast cancer cells. Breast Cancer Res Treat 1999:54:235-44.

14. Guedez L, Stetler-Stevenson WG, Wolff L, Wang J, Fukushima P, Mansoor A, et al. In vitro suppression of programmed cell death of B cells by tissue inhibitor of metalloproteinases-1. J Clin Invest 1998;102:2002-10.

15. Li G, Fridman R, Kim HC. Tissue inhibitor of metalloproteinase1 inhibits apoptosis of human breast epithelial cells. Cancer Res 1999;59:6267-75.

16. Nomura H, Fujimoto N, Seiki M, Mai M, Okada Y. Enhanced production of matrix metalloproteinases and activation of matrix metalloproteinase 2 (gelatinase A) in human gastric carcinomas. Int J Cancer 1996;69:9-16.

17. Murray GI, Duncan ME, Arbuckle E, Melvin WT, Fothergill JE. Matrix metalloproteinases and their inhibitors in gastric cancer. Gut 1998;43:791-7.

18. Mimori K, Mori M, Shiraishi T, Fujie T, Baba K, Haraguchi M, et al. Clinical significance of tissue inhibitor of metalloproteinase expression in gastric carcinoma. Br J Cancer 1997;76;531-6.

19. Yoshikawa T, Tsuburaya A, Kobayashi O, Sairenji M, Motohashi $\mathrm{H}$, Yanoma S, et al. Intratumoral concentrations of tissue inhibitor of matrix metalloproteinase-1 in patients with gastric cancer. A new biomarker for invasion and its impact on survival. Cancer 2001:91:1739-44.

20. Japanese Research Society for Gastric Cancer, editors. Japanese classification of gastric carcinoma. First English Edition. Tokyo: Kanehara; 1995.

21. Hayakawa T, Yamashita K, Kodama S, Iwata H, Iwata K. Tissue inhibitor of metalloproteinases and collagenase activity in synovial fluid of human rheumatoid arthritis. Biomed Res 1991;12: 169-73.

22. Kodama S, Iwata K, Iwata H, Yamashita K, Hayakawa T. Rapid one-step sandwich enzyme immunoassay for tisssue inhibitor of metalloproteinases. J Immunol Methods 1990;127:103-8.

23. Yoshikawa T, Tsuburaya A, Kobayashi O, Sairenji M, Motohashi $\mathrm{H}$, Noguchi Y. Is D2 lymph node dissection necessary for early gastric cancer? Ann Surg Oncol 2002;9:401-5.
24. Schrohl AS, Holten-Andersen MN, Peters HA, Look MP, Meijer-van Gelder ME, Klijn JG, et al. Tumor tissue levels of tissue inhibitor of metalloproteinase- 1 as a prognostic marker in primary breast cancer. Clin Cancer Res 2004;10:2289-98.

25. Joo YE, Seo KS, Kim HS, Rew JS, Park CS, Kim SJ. Expression of tissue inhibitors of metalloproteinases (TIMPs) in gastric cancer. Dig Dis Sci 2000;45:114-21.

26. Yano A, Nakamoto T, Hashimoto K, Usui T. Localization and expression of tissue inhibitor of metalloproteinase-1 in human urothelial cancer. J Urol 2002;167:729-34.

27. Kallakury BV, Karikehalli S, Haholu A, Sheehan CE, Azumi N, Ross JS. Increased expression of matrix metalloproteinases 2 and 9 and tissue inhibitors of metalloproteinase 1 and 2 correlates with poor prognostic variables in renal cell carcinoma. Clin Cancer Res 2001;7:3113-9.

28. Aljada IS, Ramnath N, Donohue K, Harvey S, Brooks JJ, Wiseman SM, et al. Upregulation of the tissue inhibitor of metalloproteinase-1 protein is associated with progression of human non-small-cell lung cancer. J Clin Oncol 2004;22:3218-29.

29. Nakopoulou L, Giannopoulou I, Stefanaki K, Panayotopoulou E, Tsirmpa I, Alexandrou P, et al. Enhanced mRNA expression of tissue inhibitor of metalloproteinase-1 (TIMP-1) in breast carcinomas is correlated with adverse prognosis. J Pathol 2002;197: 307-13.

30. Mori M, Mimori K, Sadanaga N, Inoue H, Tanaka Y, Mafune K, et al. Prognostic impact of tissue inhibitor of matrix metalloproteinase-1 in esophageal carcinoma. Int $\mathrm{J}$ Cancer 2000;88:575-8.

31. Fong KM, Kida Y, Zimmerman PV, Smith PJ. TIMP-1 and adverse prognosis in non-small cell lung cancer. Clin Cancer Res 1996;2:1369-72.

32. Holten-Andersen M, Christensen IJ, Nilbert M, Bendahl PO, Nielsen HJ, Brunner N, et al. EORTC-Receptor and Biomarker Group. Association between preoperative plasma levels of tissue inhibitor of metalloproteinase 1 and rectal cancer patient survival: a validation study. Eur J Cancer 2004;40:64-72.

33. Manenti L, Paganoni P, Floriani I, Landoni F, Torri V, Buda A, et al. Expression levels of vascular endothelial growth factor, matrix metalloproteinases 2 and 9 and tissue inhibitor of metalloproteinase 1 and 2 in the plasma of patients with ovarian carcinoma. Eur J Cancer 2003;39:1948-56.

34. Holten-Andersen MN, Christensen IJ, Nielsen HJ, Stephens RW, Jensen $\mathrm{V}$, Nielsen $\mathrm{OH}$, et al. Total levels of tissue inhibitor of metalloproteinases 1 in plasma yield high diagnostic sensitivity and specificity in patients with colon cancer. Clin Cancer Res 2002;8:156-64.

35. Holten-Andersen MN, Stephens RW, Nielsen HJ, Murphy G, Christensen IJ, Stetler-Stevenson W, et al. High preoperative plasma tissue inhibitor of metalloproteinase-1 levels are associated with short survival of patients with colorectal cancer. Clin Cancer Res 2000;6:4292-9.

36. Durkan GC, Nutt JE, Marsh C, Rajjayabun PH, Robinson MC, Neal DE, et al. Alteration in urinary matrix metalloproteinase-9 to tissue inhibitor of metalloproteinase- 1 ratio predicts recurrence in nonmuscle-invasive bladder cancer. Clin Cancer Res 2003;9: 2576-82.

37. Ylisirnio S, Hoyhtya M, Makitaro R, Paaakko P, Risteli J, Kinnula VL, et al. Elevated serum levels of type I collagen degradation marker ICTP and tissue inhibitor of metalloproteinase (TIMP) 1 are associated with poor prognosis in lung cancer. Clin Cancer Res 2001;7:1633-7.

38. Yoshikawa T, Tsuburaya A, Kobayashi O, Sairenji M, Motohashi $\mathrm{H}$, Yanoma S, et al. Prognostic value of tissue inhibitor of matrix metalloproteinase-1 in plasma of patients with gastric cancer. Cancer Lett 2000;151:81-6.

39. Yoshikawa T, Saitoh M, Tsuburaya A, Kobayashi O, Sairenji M, Motohashi $\mathrm{H}$, et al. Tissue inhibitor of matrix metalloproteinase-1 in the plasma of patients with gastric carcinoma. A possible marker for serosal invasion and metastasis. Cancer 1999;86:1929-35. 
40. Yukawa N, Yoshikawa T, Akaike M, Sugimasa Y, Takemiya S, Yanoma S, et al. Plasma concentration of tissue inhibitor of matrix metalloproteinase 1 in patients with colorectal carcinoma. Br J Surg 2001;88:1596-601.

41. Yukawa N, Yoshikawa T, Akaike M, Sugimasa Y, Takemiya S, Yanoma S, et al. Prognostic impact of tissue inhibitor of matrix metalloproteinase-1 in plasma of patients with colorectal cancer. Anticancer Res 2004;24:2101-5.

42. Yoshiji H, Harris SR, Raso E, Gomez DE, Lindsay CK, Shibuya $\mathrm{M}$, et al. Mammary carcinoma cells over-expressing tissue inhibi- tor of metalloproteinases- 1 show enhanced vascular endothelial growth factor expression. Int J Cancer 1998;75:81-7.

43. Cornelius LA, Nehring LC, Harding E, Bolanowski M, Welgus HG, Kobayashi DK, et al. Matrix metalloproteinases generate angiostatin: effects on neovascularization. J Immunol 1998;161: $6845-52$.

44. Yoo CH, Noh SH, Shin DW, Choi SH, Min JS. Recurrence following curative resection for gastric carcinoma. Br J Surg 2000;87: 236-42. 